\title{
2 Forschungsüberblick und Desiderate
}

Die Untersuchung verortet sich in zwei historischen Forschungsfeldern, der Wissensgeschichte und der Gefühlsgeschichte, die in den letzten 15 Jahren im Kontext der Kulturwissenschaften und der Wissenschaftsgeschichte entstanden sind. ${ }^{1}$

\subsection{Wissensgeschichte - Gefühlsgeschichte - Gefühlspolitik}

\section{Wissensgeschichte}

Der wissensgeschichtliche Ansatz entwickelte sich bereits in den 1960er Jahren aus dem soziologischen Konzept der „Wissensgesellschaft“" heraus, in welchem die Bedeutung von Wissen und Information für moderne Gesellschaften untersucht wurde. Die interdisziplinäre deutschsprachige Wissensgeschichte existiert namentlich erst seit ein paar Jahren. Dieser Forschungsansatz geht davon aus, dass Akteur^innen, Institutionen, Objekte und Medien an der Generierung, Vermittlung, Adaption, Zirkulation, Transformation und Rezeption partizipieren und die Anwendung von Wissen aktiv mitgestalten. ${ }^{3}$ Dieser breit gefasste Wissensbegriff betont die „Verflechtung gesellschaftlicher Bereiche in der Generierung, Kommunikation und Anwendung von Wissen“ und umfasst „die Gesamtheit des Wissens, das Mitglieder[n] einer Kultur [...] eigen ist und innerhalb dieser Kultur tradiert wird.“4 Dieses „an bestimmte Kulturen gebundene[...] Wissen“ könne „implizites als auch explizites Wissen umfassen“ und sowohl empirisch geltende „Fakten“, terminologische und theoretische Konstrukte als auch „kulturelle Denk-, Orientierungs- und Handlungsmuster" miteinschließen. ${ }^{5}$

Im Begriff der „Wissenskulturen“ wird mit Kultur „die Gesamtheit menschlicher Hervorbringungen“ begriffen, wobei Wissen als eine „kulturelle Leistung“ aufgefasst wird. ${ }^{6}$ Wissenskulturen sind daher ambivalente Gebilde, die „aus einer

1 Zur aktuellen Methodik- und Theoriedebatte vgl. Frevert und Nagy 2019, Mulsow und Daston 2019.

2 Vgl. Stehr 1994. Zur Wissenspolitik vgl. Stehr 2003.

3 Forschungsüberblick zur Wissenschaftsgeschichte und Wissensgeschichte vgl. Sommer u. a. 2017. Mehr zur Wissens- und Wissenschaftsgeschichte vgl. u.a. Hagner 2001, Plamper 2004, Sommer 2008, Sarasin 2011, Speich Chassé und Gugerli 2012, Sommer 2015, Sommer 2016.

4 Vgl. Müller-Wille, Reinhardt, Sommer 2017, 3.

5 Vgl. ebd.

6 Vgl. Fried und Kailer 2003, 9.

ว OpenAccess. () 2021 Isabelle Haffter, publiziert von De Gruyter. (cc) BY-NC-ND Dieses Werk ist lizenziert unter einer Creative Commons Namensnennung - Nicht kommerziell - Keine Bearbeitung 4.0 International Lizenz. https://doi.org/10.1515/9783110661439-002 
Vielzahl unterschiedlicher Akteure, Institutionen und Praktiken“ bestehen und „,in vielfältigen, wandelbaren und oft weite Distanzen überwindenden Austauschund Konkurrenz- und Machtbeziehungen stehen“.7 Die Wissenssoziologin Karin Knorr Cetina betont in ihrer Begriffsbestimmung der „Wissenskulturen“ den Aspekt der „Kultur als Praxis“, indem sie Kultur auf eine „Wissenspraxis“ bezieht. ${ }^{8}$ Unter „Wissenskultur“9 versteht sie „diejenigen Praktiken, Mechanismen und Prinzipien, die, gebunden durch Verwandtschaft, Notwendigkeit und historische Koinzidenz, in einem Wissensgebiet bestimmen, wie wir wissen, was wir wissen. Wissenskulturen generieren und validieren Wissen."10

Wissenskulturen aus dem Theaterbereich bilden einen Forschungsschwerpunkt, der in den letzten Jahren, u. a. in der Literatur- und Theaterwissenschaft, Einzug gehalten hat. Stefan Hulfeld untersuchte die Theatergeschichtsschreibung als kulturelle Praxis vom 16. bis ins 20. Jahrhundert und fragte nach Zielen und Zwecken, unter denen Wissen über Theater gesammelt, ergänzt und verbreitet worden war. Für die vorliegende Untersuchung sind insbesondere die von Hulfeld untersuchten ambivalenten Abgrenzungsstrategien aufschlussreich (einerseits der Theaterhistoriographie von der Germanistik, andererseits der Theaterwissenschaft im Sinne des Berliner Institutsgründers Max Herrmanns zur Theaterhistoriographie). ${ }^{11}$ Beate Hochholdinger-Reiterer zeigte die wissenschaftshistorischen Beziehungsgeflechte zwischen Wissen, Geschlecht, Theater und Politik, u.a. in den theaterwissenschaftlichen Schriften Heinz Kindermanns in Österreich während der NS-Zeit, auf. ${ }^{12}$

\section{Gefühlsgeschichte}

Spätestens seit den 1990er Jahren befassen sich Vertreter*innen der Affect Studies unter dem Schlagwort eines emotional turns mit Emotions- und Affekttheorien. ${ }^{13}$ Die geisteswissenschaftliche Auseinandersetzung mit Emotionen und ästheti-

7 Vgl. Müller-Wille, Reinhardt, Sommer 2017, 3f, 10. Vgl. Neumann 2013.

8 Vgl. Knorr Cetina 2002, 19. Vgl. auch Sandkühler 2007.

9 Vgl. Knorr Cetina 2002, 11.

10 Vgl. ebd.

11 Vgl. Überwindung normativer Theaterhistoriographie. In: Hulfeld 2007, 271-281. Zu den Problemen der Theatergeschichtsschreibung vgl. Bayerdöfer 1990. Mehr dazu s. Kap. 4.1.

12 Vgl. Hochholdinger-Reiterer 2014. Mehr zur NS-Theaterwissenschaft s. Kap. 2.4.

13 Vgl. Anz 1999, Anz 2006. Zu Affect Theory und Studies vgl. Gregg und Seigworth 2010, Baier u. a. 2014. 
scher Erfahrung von Kunst, Literatur, Musik- oder Theateraufführungen reicht jedoch bis in die Antike zurück. ${ }^{14}$

Der Forschungsbereich „Geschichte der Gefühle“ des Max-Planck-Instituts für Bildungsforschung (MPIB) in Berlin vertritt aus einer emotionshistorischen Perspektive die These, dass Gefühle oftmals in Mischformen auftreten, die andere Gefühle in ihre Praktiken miteinbinden und dadurch zu neuen Erscheinungsformen und Bedeutungskontexten gelangen. ${ }^{15}$ Die Gefühlsgeschichte fragt u.a. nach den historischen Legitimations- und Stabilisierungsstrategien, in welchen Gefühle politische Machtverhältnisse, Ideologien und „moralische Ökonomien“ (,moral economies“) ${ }^{16}$ beeinflussen und lenken, wie Ute Frevert in ihrem Forschungsprojekt „Die Macht der Gefühle: Politische Kommunikation zwischen oben und unten“ zeigt. ${ }^{17}$ Thomas Rohringer beleuchtet die „Arbeitsfreude“ in der moralischen Ökonomie der Reintegration von Kriegsgeschädigten während des Ersten Weltkriegs. ${ }^{18}$ Juliane Brauer untersuchte beispielweise den Einfluss von Musizieren und Singen in NS-Konzentrationslagern oder als Erziehungsinstrument bei der DDR-Jugend unter Berücksichtigung affirmativer Gefühlszustände wie „Glück“, Freude und Wohlbefinden. ${ }^{19}$

Historische Studien beschäftigen sich bereits seit Jahren (mehr oder weniger explizit) mit Gefühlen im Nationalsozialismus. Ein Beispiel ist der affektive Massenspektakelcharakter der Nürnberger „Reichsparteitage“, welcher von der Regisseurin Leni Riefenstahl 1933 und 1934 in propagandistischen Dokumentaraufnahmen filmisch umgesetzt wurde. ${ }^{20}$ Das Erlebnis solcher nationalsozialistischer Propagandaveranstaltungen wurde von Zeitzeugen oftmals als Glücksrausch, Euphorie und Ekstase-Erfahrung beschrieben. ${ }^{21}$ Semantiken und Trabanten von „Glück“ wurden sowohl in Interviewaussagen von Zeit-

14 Vgl. Frevert 2009, 190. Vgl. Landweer 2008. Auf die antike Emotionstheorie bei Aristoteles wird in Kap. 7 Bezug genommen.

15 Vgl. Laukötter o.J., Frevert 2009, 191f., Hitzer 2011, Hitzer und Gammerl 2013.

16 Untersuchungen moralischer Ökonomien aus emotionshistorischer Perspektive, vgl. Frevert 2019b.

17 Zum Forschungsprojekt: https://www.mpib-berlin.mpg.de/forschung/forschungsbereiche/ge schichte-der-gefuehle/forschungsfelder/buerger-und-nation/die-macht-der-gefuehle, 22.11.2020. Vgl. Frevert 2020.

18 Vgl. Rohringer 2019.

19 Vgl. Brauer 2015, Brauer 2016, Brauer 2019, Brauer 2020.

20 Besondere Bekanntheit erlangte der NS-Propagandafilm „Triumph des Willens“ (1935) aufgrund seiner filmtechnischen und -ästhetischen Qualität. Mehr zu Medien und NS-Propaganda vgl. Loiperdinger 1987. Vgl. auch Reichel 2006, Zimmermann 2007, Schrader 2008, Heidenreich 2010, Zalfen und Müller 2012.

21 Vgl. Brockhaus 1997, Klimó und Rolf 2006, Herbst 2010. 
zeug^innen als auch in Quellen der NS-Parteifunktionäre (z.B. in Joseph Goebbels (1897-1945) Tagebüchern oder in „Stimmungsberichten“22) oder in zeitgenössischen Publikationen faschistischer, antifaschistischer und NSsympathisierender Wissenschaftler ${ }^{\star}$ innen genannt. Diese sind aber bisher kaum systematisch unter einer explizit emotions- und wissenshistorischen Perspektive untersucht worden. ${ }^{23}$

Studien aus der Wissenschaftsgeschichte und der Wissenssoziologie $\mathrm{zu}$ Wissenskulturen von Gefühlen im Allgemeinen sind erst vereinzelt erschienen. ${ }^{24}$ Seitens der Theatergeschichte untersuchte beispielsweise die Theaterwissenschaftlerin Doris Kolesch die „höfische Emotionalität“ zur Zeit Louis XIV., indem sie die historische Bedeutung ,des Theaters“als „emotionale Gefühlsmaschinerie“ in der politischen Machtkonstellation des Absolutismus erforschte. ${ }^{25}$ Der Theaterwissenschaftler Matthias Warstat beschäftigte sich mit den theaterästhetischen Wirkungsdimensionen in den Heils- und Krisendiskursen von den Avantgarden bis in die Gegenwart. ${ }^{26}$

Soziologen ${ }^{\star}$ innen, Kulturanthropolog*innen, Politikwissenschaftler*innen und Historiker^innen befassen sich gleichermaßen mit Manifestationen von Gefühlen wie Wut, Angst oder Hass, die im Kontext sozialer und politischer Protestbewegungen, beispielsweise im Kontext des „Kalten Krieges“ und der 1968erund 1980er- Revolten, auftraten. ${ }^{27}$ Ein weiteres Beispiel aus dem Bereich der Gefühlsgeschichte ist der Buchband über „Gefühlswissen“ ${ }^{28}$, begriffen als eine „lexikalische Spurensuche“, in welchem mit Hilfe von Lexika und Enzyklopädien unterschiedliche Gefühlsdiskurse in westlichen Gesellschaften vom 18. bis zum frühen 21. Jahrhundert untersucht wurden. Der Sammelband geht von der An-

22 Vgl. Boberach 1984.

23 Historische Forschungen zum NS-Regime, Antisemitismus, Rassismus, aber auch Nationalismus und (Post-)Kolonialismus, in denen Emotionen eine explizite Analysekategorie darstellen, konzentrieren sich, dem Untersuchungsgegenstand entsprechend, oft auf negative Emotionen wie z. B. Hass, Angst, Wut, vgl. u.a. Kershaw 1999, Reichel 2006, Gross 2010, Marks 2011a, Jensen und Schüler-Springorum 2013, Bialas 2014, Rauschenberger, Konitzer und Fritz Bauer Institut 2015, Frevert 2015, Parkinson 2015, Holmes 2016, Hamann 2016, Wodak 2016.

24 Vgl. Jensen und Morat 2008, Daston 2014, Nay 2014, Frevert 2017a. Eine Wissenschaftsgeschichte, beispielsweise zur psychologischen Emotionsforschung, ist noch ausstehend. Vgl. Frevert 2011, 10, Fußnote 3. Vgl. Jensen 2019.

25 Vgl. Kolesch 2006.

26 Vgl. Warstat 2011.

$27 \mathrm{Zu}$ sozialen (Protest-)bewegungen und Emotionen vgl. Goodwin 2001, Goodwin und Jasper 2004, Klandermans 2007, Tanner 2008, Flam und King 2008, Roose und Dietz 2016, Knud, Steen und Institut für soziale Bewegungen, Ruhr-Universität, Bochum 2016. Zum Aktualitätsbezug der Machtbeziehungen aus Politik, Gefühlen und Bevölkerung s. Kap. 18.

$28 \mathrm{Zu}$ Gefühlswissen vgl. Frevert 2011. 
nahme aus, dass Gefühle erlernbar und das Wissen über Gefühle, das sog. „Gefühlswissen“, konstruiert sei. ${ }^{29}$

In der vorliegenden Untersuchung sollen, ausgehend vom Konzept des Gefühlswissens, Wandel, Kontinuitäten und Ambivalenzen von „Glück“ als ein historischer Quellenbegriff untersucht werden. Dabei handelt es sich in mehrfacher Hinsicht um ein Forschungsdesiderat, was in diesem Kapitel aufgezeigt wird.

\section{Gefühlspolitik}

Der Begriff „Gefühlspolitik“30 wird in der vorliegenden Untersuchung einerseits in Anlehnung an Ute Freverts emotionshistorische Forschungen verwendet, um eine „Politik mit Gefühlen“ zu beschreiben. ${ }^{31}$ Der Begriff stammt aus dem 19. Jahrhundert und wurde von Otto von Bismarck (1815-1898) verwendet, um in abschätziger Weise über jene politischen Romantiker^innen zu sprechen, die „Politik mit Moral und/oder Gefühl“ vertauschten. ${ }^{32}$ Frevert verwendet den Begriff der „Gefühlspolitik“ jedoch nicht in seiner historischen Semantik. „Gefühlspolitik“ sei „eine Politik, die Gefühle inszenierte, adressierte, erzeugte und in Dienst nahm“, um auf nationaler und/oder internationaler Ebene die Machtbeziehungen zwischen Staaten und Bevölkerungen zu verbessern. ${ }^{33}$

Im Unterschied zu Frevert nimmt die vorliegende Arbeit für ihre theaterhistorische Untersuchung einer „Gefühlspolitik“ vom theaterwissenschaftlich geprägten Begriff der Inszenierung als einen Analysebegriff Abstand, um methodi-

29 Vgl. Frevert 2011. Vgl. auch Jensen und Morat 2008, Dror u. a. 2016. Zur „Gefühlspolitik“ bzw. $\mathrm{zu}$ historischen, anthropologischen, politikwissenschaftlichen und politik-philosophischen Forschungen über Emotionen, Politik und Nation vgl. u. a. Hughes 1983, American 1990, François, Siegrist und Vogel 1995, Goodwin 2001, Mitchell 2009, Ciompi 2011, Hoggett und Thompson 2012, Heidenreich und Schaal 2012, Nussbaum 2013, Demertzis 2013, Ahmed 2014, Nielsen 2015, Korte 2015.

30 Zur „Gefühlspolitik“ bzw. zu historischen, anthropologischen, soziologischen, politikwissenschaftlichen und politik-philosophischen Forschungen zu Emotionen, Politik und Nation, vgl. u. a. Hughes 1983, American Anthropological Association 1990, François, Siegrist und Vogel 1995, Goodwin 2001, Stearns 2006, Mitchell 2009, Ciompi 2011, Hoggett und Thompson 2012, Frevert 2012, Heidenreich und Schaal 2012, Nussbaum 2013, Demertzis 2013, Ahmed 2014, Nielsen 2015, Korte 2015.

31 Vgl. Frevert 2012, 16.

32 Vgl. ebd.

33 Vgl. ebd., 17. 
sche und theoretische Missverständnisse zu vermeiden. ${ }^{34}$ „Gefühlspolitik“ wird in der vorliegenden Arbeit als wissenshistorischer Begriff verwendet. Bereits der deutsche Historiker Siegfried August Kaehlers (1885-1963) benutzte den Begriff „Gefühlspolitik“ in Referenz an Bismarck zur Erläuterung der Divergenz zwischen nationalsozialistischer „Realpolitik“ und „Gefühlspolitik“. ${ }^{35}$ Als Beispiel nennt er u. a. die emotionspolitische Annexionsstrategie im Falle Österreichs im Jahr 1938. Kaehler erklärt sich den Erfolg der affirmativen Gefühlspolitik der Nationalsozialisten als Folge eines nach dem Zerfall des Habsburgerreiches entstandenen „Minderwertigkeitsgefühls vieler Deutsch-Österreicher“:

„In enger Verbindung mit dieser auf binnenvölkische Ziele ausgerichteten Behandlung außerpolitischer Fragen steht die Tatsache, daß die unter Bereitstellung stets wachsender Machtmittel geführte nationalsozialistische Aussenpolitik [...] trotz ihres machtpolitischen Gehabes alles andere war, als Realpolitik im Sinne Bismarcks. Vielmehr war sie Gefühlspolitik, deren Wurzeln im innerpolitischen Machtverfall des Habsburgerreichs [...] und der aus diesem Verfall erwachsenden Minderwertigkeitsgefühle vieler Deutsch-Österreicher zu suchen sind. Daran ändert nichts den Umstand, daß der Anschlußgedanke in weiten Kreisen des national empfindenden Deutschlands einschließlich der sozialdemokratischen Partei diesseits wie jenseits der Reichgrenze freudig begrüßt und seine Verwirklichung lebhaft gewünscht wurde. “36

Die „völkische“ Forderung Hitlers nach mehr „Lebensraum“, die „geopolitische“ „Vereinigung aller Deutschen“ sowie der „Gleichberechtigungsgedanke“, wie er ihn in einem Interview gegenüber dem französischen Korrespondenten Pierre Huß am 16.1.1935 geäußert hatte, basierten, neben militärstrategischen Absichten, nach Außen auf einer emotionspolitischen Argumentation. Diese besage, nach Hitler, dass die nationale Souveränität Deutschlands als auch Österreichs durch

34 Fischer-Lichte versteht unter „Inszenierung“ den „Vorgang der Planung, Erprobung und Festlegung von Strategien [...], nach denen die Materialität einer Aufführung performativ hervorgebracht werden soll, wodurch zum einen die von ihr hervorgebrachten Ereignisse als gegenwärtige in Erscheinung treten und zum anderen eine Situation geschaffen wird, die Frei- und Spielräume für nicht-geplante, nicht-inszenierte Handlungen, Verhaltensweisen und Ereignisse eröffnet“, Fischer-Lichte 2014a. Der Aspekt des Ereignisses als gegenwärtige Erscheinung schließt eine theaterhistorische Betrachtung per definitionem aus. Ähnliche Feststellungen machte bereits der Theaterwissenschaftler Max Herrmann 1931 in „Das theatralische Raumerlebnis“, vgl. u.a. Herrmann 1914, Herrmann 1981, Herrmann 1998a. Mehr zu Herrmann vgl. Corssen 1998, Hollender 2013, Dörschel und Warstat 2018.

35 Vgl. Kaehler, „Realpolitik, Gefühlspolitik, Gleichberechtigung in der NS-Aussenpolitik“ [Vortragsmanuskript, ca. 1951] Nachlass Siegfried August Kaehler, Signatur Cod.Ms.S A. Kaehler 4:15,3, SUB Göttingen.

36 Kaehler, „Realpolitik, Gefühlspolitik, Gleichberechtigung in der NS-Aussenpolitik“ [Vortragsmanuskript, ca. 1951], 7, SUB Göttingen. 
die Beschlüsse des Versailler Vertrags diffamiert worden seien. ${ }^{37}$ Hitlers ,affektbetonte“ Forderungen hätten zunächst den Anschein einer „liberalen“ Auffassung bezüglich einer „völkerrechtlich“ „,moralische[n]“ Gleichberechtigung“ vermittelt (ein Denken, das Hitler jedoch verabscheute). ${ }^{38}$ Dieser diplomatische Schachzug einer internationalen Befriedungsstrategie sei jedoch mit den Jahren immer mehr einer „völkischen“ Gefühlspolitik der außenpolitischen Fremd- und innenpolitischen „Selbsttäuschung“ gewichen. ${ }^{39}$ Vom „Machtrausch befallen“ ${ }^{“ 40}$, sei Hitlers Gefühlspolitik militärstrategischen Expansionsfantasien verfallen.

Ein weiteres entscheidendes Element der NS-Gefühlspolitik sei das rassistisch konstruierte Zusammengehörigkeitsgefühl, welches Hitler u.a. in der Umbenennung Berlins in „Germania“ zu demonstrieren versucht habe:

„Denn der Name Germania für die Reichshauptstadt in ihrer neuen repräsentativen Form sei geeignet, trotz räumlicher Entfernung zwischen jedem Angehörigen des [der] germanischen Rasse und dieser Hauptstadt ein Gefühl der Zusammengehörigkeit zu erzeugen.““11

Vor dem Hintergrund des aktuellen Forschungsstands zu Gefühlen, Politik und Krieg $^{42}$ im interdisziplinären Feld der Affect Studies und der Emotionsgeschichte begreift die vorliegende Untersuchung Gefühle weder als eine ausschließlich intellektuell noch als eine körperlich empfundene Reaktion auf äußere Reize, sondern als einen historisch ambivalenten Quellenbegriff. ${ }^{43}$

\subsection{Glückswissen: „Glück“ als Untersuchungsgegenstand der Kulturwissenschaften}

Wie rekonstruiert die Sprachwissenschaft etymologisch den deutschen Begriff von „Glück“? Laut Brockhaus liegt die Eigenheit des deutschen Wortes „Glück“ in seiner Ambivalenz: Im Altgriechischen, Lateinischen, Französischen oder Engli-

37 Ebd., $10 \mathrm{f}$.

38 Vgl. ebd., 13.

39 Vgl. ebd., 11.

40 Vgl. ebd., 14.

41 Kaehler, „Realpolitik, Gefühlspolitik, Gleichberechtigung in der NS-Aussenpolitik“ [Vortragsmanuskript, ca. 1951], [angeheftete Notiz]. Kaehler verweist hier auf „Picker, S. 98“ und meint damit vermutlich „Hitlers Tischgespräche im Führerquartier 1941-1941“ Ersterscheinung 1951, vgl. Picker 1951, 98.

42 Forschungen zu Ehre, Scham, Politik und Krieg vgl. u. a. Frevert 2001, Brunner 2010, Marks 2011b, Frevert 2013a, Heidgen 2013, Aschmann 2013, Gertiser 2015, Rosenwein 2016.

43 Vgl. Brauer 2015, $167 \mathrm{ff}$. 
schen wird zwischen der „Gunst der Umstände“ (altgriech. eutychía; lat. fortuna; frz. fortune; engl. luck) und dem Gefühl und der Empfindung von „Glück“ und der Glückseligkeit unterschieden (altgriech. eủdaimonía; lat. beatitudo; frz. bonheur; engl. happiness). Im Deutschen existiert jedoch nur ein Begriff: „Glück“ (mhd. gelücke [Geschick, Zufall], heil [glückl. Zufall, Gesundheit] und salig [gut, gesegnet, selig]). ${ }^{44}$ Die Mehrdeutigkeit des Glücksbegriffs widerspiegelt sich in den seit der Antike geführten Glücksdiskursen. Philosoph*innen und Literaturwissenschaftler^innen haben insbesondere die Dichotomie zwischen „Glück“ und „Unglück“ und die Differenz zwischen dem momenthaften Gefühl des „Glücklichseins“, dem „Glück haben“ als schicksalhaftem oder zufälligem Zustand und dem zukünftigen (zielgerichteten) „Glück“ als Vorstellung hervorgehoben. ${ }^{45}$

„Glück“ wird von der interdisziplinären Glücksforschung, die ihrerseits ein Teil der Wissenskulturen ist, seit den 1980er Jahren erforscht. ${ }^{46}$ Kulturen von „Glück“ sind nicht nur zeitgenössische Erscheinungen, sondern wurden von Darrin McMahon historisch bis in die Antike zurückverfolgt. ${ }^{47}$ Die Emotionshistorikerin Frevert beleuchtet in einem Aufsatz Glückswissen anhand des Märchens „Hans im Glück“, indem sie die Moral des Märchens im Kontext moralischer Ökonomien analysiert. ${ }^{48}$ Der Sammelband „Petrified Utopia. Happiness Soviet Style“ untersucht historische Konzepte von „Glück“ in der Sowjetkultur für die Bereiche Literatur, Kunst, Film und Architektur, jedoch unter Auslassung von Theater und Wissenschaft. ${ }^{49} \mathrm{Zu}$ amerikanischen Kulturen von „Glück“ verfasste Jackson Lears eine historische Studie, in welcher er den Fokus auf ökonomische Erscheinungen wie das Glücksspiel legt. ${ }^{50}$

Stephanie Kleiner historisierte und rekonstruierte erstmals, gemäß dem aktuellen Wissensstand der Autorin, „spezifische Logiken bzw. Praktiken der Ge-

44 Vgl. Brockhaus 2006, 84-87, 84.

45 Vgl. u. a. Aristoteles 1982, zu Glück: 19, 21, 27, 35, 39, 41, 57, „Freude an der Nachahmung“: 11, im Nachwort, Glück: 158, 160, Vergnügen: 161 ff., 165f., Tanzer 2011, 17 f., Thomä 2011.

46 Häufig zitierte Autoren aus Soziologie, Psychologie, Religions-, Literatur-, Politik-, und Wirtschaftswissenschaft sind u.a. Csikszentmihalyi 1985, Bellebaum, Barheier und Institut für Glücksforschung 1997, Jordan, Bellebaum und Institut für Glücksforschung 1998, Bellebaum und Barheier 1998, Bellebaum 2002, Bellebaum 2005, Layard 2005, Bellebaum und Herbers 2006, Frey und Frey Marti 2010.

47 Vgl. McMahon 2006; vgl. auch Poser 2017.

48 Vgl. Frevert 2019d.

49 Vgl. Balina und Dobrenko 2011. Vgl. auch Plamper 2012b. Zu „Glück“ in der Literaturgeschichte und dem „Eudaemic Turn“ in der Literaturwissenschaft vgl. u.a. Bellebaum und Muth 1996, Pye 2002, Tanzer 2011, Pawelski und Moores 2013, Caston und Kaster 2016.

50 Vgl. Lears 2003. 
nerierung von ,Glückswissen““ anhand nicht näher definierter „Konzepte individuellen und kollektiven Glücks“ im Bereich der Wissens- und Sozialgeschichte. ${ }^{51}$

\subsection{Lebensführung, Ratgeber, Psychotechnik, „Kraft durch Freude“, 1933-1945}

\section{Lebensführung in der Ratgeberliteratur}

Das 20. Jahrhundert kann als „das Zeitalter der Beratung“52 gelten. Das dynamische Wechselspiel zwischen Angebot und Nachfrage fand im Zuge moderner Gruppen- und Identitätsbildung, Selbstoptimierungs- und Selbstdisziplinierungsstrategien statt. ${ }^{53}$ Das Erlangen einer guten „Lebensführung“554 wurde mit

51 Vgl. Projekt „Glückswissen. Zur Geschichte einer Reflexionsform gelingender Integration im 20. Jahrhundert“ (2011-), https://www.exzellenzcluster.uni-konstanz.de/2724.html, 17.01. 2020.

52 Vgl. Macho 1999, 29.

53 Zur Zeitgeschichte des Selbst betreffend Selbsttechnologien, Therapeutisierung, Politisierung, Emotionalisierung vgl. Eitler und Eberfeld 2015. Zur Ratgeberliteratur 1900 -1940 vgl. Kleiner und Suter 2015.

54 Der Begriff der „Lebensführung“ (L.) wurde u.a. von Max Webers religionssoziologischer Schrift „Die protestantische Ethik und der Geist des Kapitalismus“ (1905) über die protestantische Berufsethik als sittliche Pflicht geprägt. Weber stellt einen Zusammenhang zwischen einer „methodisch-rationalen bürgerlichen Lebensführung“, dem modernen „rationalen Betriebskapitalismus“ und der Bürokratie her, vgl. Band 2000, 402. Die L. seiner Zeit war für Weber von Zwängen bestimmt, welche die Erwerbsarbeit im Zusammenspiel mit einer asketischen Berufsmoral und der jeweiligen Klassenzugehörigkeit hervorriefen. „Die Lebensinhalte überhaupt werden nicht auf Personen, sondern auf ,sachliche‘ rationale Zwecke ausgerichtet, die Caritas selbst ein sachlicher Armenpflegebetrieb zur Mehrung des Ruhmes Gottes. Und da der Erfolg der Arbeit das sicherste Symptom ihrer Gottwohlgefälligkeit ist, so ist der kapitalistische Gewinn einer der wichtigsten Erkenntnisgründe, daß der Segen Gottes auf dem Geschäftsbetrieb geruht hat. Es ist klar, daß sich dieser Lebensstil mit der für die ,bürgerliche‘ Erwerbsarbeit [...] - Geldgewinn und Besitz nicht als Selbstzweck, sondern als Maßstab von Tüchtigkeit - am intimsten [sic] berührt und geradzu deckt: die Einheit des religiösen Postulats mit dem für den Kapitalismus günstigen bürgerlichen Lebensstil ist erreicht.“ (Vgl.Weber 2009a, 207 f.) Weber verwendete den Begriff der L. synonym zu „Lebensstil“ (LS) und bezog sich u. a. auf den Zeitgenossen Georg Simmel. Dieser stellte u. a. in seiner „Philosophie des Geldes“ (1900) einen modernen, städtischen Lebensstil fest. Dieser zeichnete sich durch Sachlichkeit, Unverbindlichkeit und Indifferenz aus und stand dem sozialen, intellektuellen und geldwirtschaftlichen Freiheitsgewinn und den vielfältigen Möglichkeiten zur individuellen Identitätsentwicklung in der temporeichen und reizüberflutenten Großstadt ambivalent gegenüber. Vgl. Simmel 1930, Simmel 2008. Mehr zu Simmels Lebensstilbegriff vgl. Müller 2018. Pierre Bourdieu entwickelte den „Habitus“-Begriff u.a. in Bezug auf Webers L.Konzept, vgl. Bourdieu 2016. Vgl. Band 2000, 402. Mehr zur Forschung bzgl. „Lebensführung“ vgl. Schwenk 1996. Mehr zu Weber vgl. Rehberg 2014. 
Hilfe der Ratgeberliteratur im westlichen Buchmarkt als ein kulturelles Massenphänomen kommerzialisiert. Gegenwärtig setzt sich der Trend einer Wissenspraktik der Beratung, neben der psychologischen und medizinischen Beratung, auch als Unternehmens-, Verkaufs- und Rechtsberatung ${ }^{55}$ in verschiedenen analogen und digitalen Wissens-, Diskurs- und Kommunikationsräumen fort. ${ }^{56}$ Doch die Wissensgeschichte der Ratgeberpraktik auf der Grundlage der Druckschrift reicht bis zur Erfindung des Buchdrucks ins 15. Jahrhundert zurück. Das damals neue Buchmedium ergänzte eine bereits existierende mündliche Ratgebertradition. Das Ratgeber- oder Sachbuch basierte in seinen Anfängen auf einem abgedruckten Lehrgespräch, Exempel, Enzyklopädie- oder Lexikoneintrag oder einer in Sachprosa verfassten Glücks- oder Unglücksgeschichte. ${ }^{57}$ Seit dem 18. Jahrhundert hat sich das Ratgebermedium vervielfältigt. Ratgeber erschienen in Kalendern, moralischen Wochenzeitschriften, Frauenzeitschriften und seit dem 19. Jahrhundert in Illustrierten, Zeitungen und Magazinen. ${ }^{58}$ Im 20. Jahrhundert kamen beratende Rundfunksendungen, Telefongespräche, Fernsehsendungen und seit den 1990er Jahren digital und multimedial vernetzte Beratungsseiten im Internet hinzu. ${ }^{59}$

Der Untersuchungszeitraum 1933-1945 kann noch immer als Forschungsdesiderat gelten, obschon sich jüngst Einzelstudien, wie jene Lu Seegers, mit der

55 Vgl. Niehaus und Peeters 2014.

56 Think Tanks wären ein Beispiel für zukunftsgerichtete Wissensorte der (post-)modernen Beratungsgesellschaft, die während des Kalten Krieges entstanden sind und heute als interdisziplinäre Institutionen zur Unternehmensberatung eine bedeutsame Schaltstelle zwischen wissenschaftlichem „Wissen“ und Beraterwissen darstellen. Vgl. Pias und Vehlken 2010.

57 Vgl. Messerli 2010, $30 \mathrm{f}$.

58 Recherchierte Beispiele: Der Mensch, eine moralische Wochenschrift (1756). Zwölfter Theil, Halle: Johann Justinus Gebauer; Hülfreich, Erdmuthe (1797): Der Erdmuthe Hülfreichinn Wirthschaftskalender für Hausmütter, in welchem ihre Geschäfte in der Küche, im Garten, im Felde, im Viehstalle, beym Geflügel, und auch andere häusliche Geschäfte durch alle Monathe des Jahres angezeigt und erkläret werden, Wien: Aloys Doll; Klencke, Hermann (1869): 2 Bände in 1 Band. 1. Kosmetik oder menschliche Verschönerungskunst auf Grundlage rationeller Gesundheitslehre. 2. Der Frauenarzt. Lehrbuch für das weibliche Geschlecht über dessen Gesundheits- und Heilpflege, Leipzig: Eduard Kummer.

59 Vgl. Messerli 2010, 31. Zur (Glücks-)Ratgebertradition 1945 bis heute vgl. u. a. Duttweiler 2007, Kleiner und Suter 2018. Die staatlichen Fernsehsender ARD und Schweizer Fernsehen (SRF) haben beispielsweise auf ihren Homepages (ratgeber.ard.de) exklusive Ratgeberseiten mit eigens produzierten Videoclips und Links zu Dokumentarfilmen, Fernsehsendungen, Podcasts und Blogeinträgen auf Social Media Plattformen (Instagram, Twitter, etc.) eingerichtet. Die SRF-Rubriken lauten: „Medizin“, „Digital“, „Konsum“, „Fit \& Gesund“, „Garten“, „Trick 77“, https:// www.srf.ch/sendungen/ratgeber/uebersicht, 12.02.2019. 
Ratgeberliteratur im NS-Regime auseinanderzusetzen beginnen. ${ }^{60}$ Ein transnationaler Vergleich zwischen NS-Deutschland und der Schweiz, wie er in Ansätzen in der vorliegenden Untersuchung unternommen wird, stellt einen Einzelfall dar. ${ }^{61}$

\section{Psychotechnik in der Arbeitswissenschaft}

Bereits zu Beginn des 20. Jahrhunderts kam es zu einer Verbindung zwischen sozialen Praktiken und Institutionen wissenschaftlicher Expertise. Dabei wurde das „Selbst“ als ein mittels psychologischen Wissens und Techniken immer stärker zu beratendes und zu therapeutisierendes Subjekt wahrgenommen und in einen gesellschaftspolitisch fragilen Kontext aus Arbeit, Kapitalismus und Staat gestellt. ${ }^{62}$ Glückswissen besaß bei den psychologischen Praktiken zur Fremd- und Selbsttherapierung des Subjekts eine entscheidende Bedeutung. ${ }^{63}$ Ein einschlägiges Beispiel von angewandtem Glückswissen war die sogenannte „Psychotechnik“64, die 1903 vom Arbeitspsychologen William Stern (1871-1938) erstmals begrifflich erfasst wurde. ${ }^{65}$

Die Erfahrung der leistungsorientierten Rationalisierung und Optimierung des Soldatenkörpers im Ersten Weltkrieg und die demokratischen Umstrukturierungsmaßnahmen der Industriebetriebe in den 1920er Jahren legten den Grundstein für eine europaweite Rezeption der Forschungsresultate der Arbeitswissenschaft, einer neuen Fachdisziplin der angewandten Psychologie. $\mathrm{Zu}$ den Methoden und Techniken der Arbeitswissenschaft zählten Zeit-, Bewegungs- und Ermüdungsstudien, wie die ergonomischen Berechnungen des Kraftaufwands zur optimalen (statt maximalen) Produktivität. ${ }^{66}$ Eine der einflussreichsten Methoden der Arbeitswissenschaft waren die Berufseignungstestverfahren der Psychotechnik. Diese Methoden wurden im Nationalsozialismus weiter ausgebaut. Insbe-

60 Vgl. Seegers 2003, Seegers 2015, Seegers 2018, Seegers 2019. Mehr Literaturhinweise s. Kap. 8. 61 Zur Schweiz vgl. u. a. Bubenhofer 2008. Mehr Literaturhinweise s. Kap. 8.

62 Zur (Wissenschafts-)geschichte des „Selbst“ zu Beginn des 20. Jahrhunderts, vgl. u. a. Foucault 2001, Eghigian, Killen und Leuenberger 2007, Maasen 2011, Jensen und Tändler 2012, Eitler und Eberfeld 2015, Senne und Hesse 2019.

63 Auch die aktuelle psychologische Glücksforschung beruht im Fall der Positive Psychology auf diesem Konzept. Eine kritische Studie zum Phänomen der „Happycracy“ vgl. Cabanas und Illouz 2018. Vgl. auch Freitas 2017.

64 Zur Wissenschaftsgeschichte der Psychotechnik vgl. u.a. Jaeger und Staeuble 1981, Drunen, Strien und Haas 2004, Patzel-Mattern 2010, Rabinbach 2018.

65 Vgl. Stern 1903.

66 Vgl. Giese 1930, 3622. 
sondere im Bereich der „Wehrmacht“ wurde die „Menschenauslese“ im wissenshistorischen Kontext der „NS-Rassenpolitik“ um eine „rassenhygienische“ Dimension erweitert. ${ }^{67}$

\section{Forschungsdesiderat: Glückswissen und die NS-Arbeitsmoral „Kraft durch Freude“}

Zahlreiche Forschungen haben sich mit der Freizeitorganisation „Kraft durch Freude“68, mit Aspekten der „Arbeitsfreude“69, mit Orten der Vergemeinschaftung $^{70}$ innerhalb der „Volksgemeinschaft“71 sowie der NS-Arbeitsmoral und deren mehr oder weniger ,erfolgreichen' Anwendungsbereichen befasst. ${ }^{72}$ Bis heute wurde jedoch von der Forschung die wissenshistorische Konstruiertheit von „Glück“ im Kontext einer Wissenszirkulation im Bereich der Ratgeberliteratur, der Arbeitswissenschaft und der NS-Arbeitspsychologie „Kraft durch Freude“ (aus einer vergleichenden Perspektive) nicht in den Blick genommen.

Vor dem Hintergrund des aktuellen Forschungsstands liegt der Erkenntnisgewinn der vorliegenden Arbeit darin, dass eine ambivalente Kontinuität von transnationalem Glückswissen nach 1933 am Beispiel der NS-Arbeitsmoral „Kraft durch Freude“ aufgezeigt werden kann. Erstmals sollen Gemeinsamkeiten und Unterschiede einer Politik von ,Glückskulturen' am transnationalen Beispiel der Ratgebergeberkultur im Kontext der Psychotechnik und einer „rassenhygienischen“ NS-Arbeitspsychologie „Kraft durch Freude“ herausgearbeitet werden.

67 Zur Psychotechnik im Nationalsozialismus vgl. u.a. Geuter 1984, Hachtmann 1989, Raehlmann 2005, Killen 2007, Uhl 2012, Stiegler 2016, Guski-Leinwand 2017, Aesthetic of Production in the Third Reich. In: Rabinbach 2018, 125-152.

68 Vgl. u. a. Baranowski 2007.

69 Vgl. u.a. Campbell 1989.

70 Vgl. u. a. Swett, Ross und d'Almeida 2011.

71 Vgl. u. a. Bajohr und Wildt 2009.

72 Die folgende Auswahl beschränkt sich auf den Entstehungszeitraum der vorliegenden Dissertation Mitte 2013 bis April 2020 und deren unmittelbare Relevanz für die vorliegende Untersuchung: Howind 2013, Kundrus und Steinbacher 2013, Reeken und Thießen 2013, Wildt und Kreutzmüller 2013, Brockhaus 2014, Buggeln und Wildt 2014, Reinicke u. a. 2014, Steber und Gotto 2014, Wildt 2014a, Donauer 2015, Föllmer 2016, Timpe 2016, Süß und Thießen 2017, Süß 2017, Timpe 2017, Frei 2018, Schmiechen-Ackermann u. a. 2018, Wildt 2019, Harvey u. a. 2019. 


\subsection{Theaterpolitik, Theaterpraxis, Theaterwissenschaft, 1933-1945}

„Glück“ als ein theaterhistorischer Untersuchungsgegenstand einer affirmativen Gefühlspolitik ist im Rahmen eines transnationalen Vergleichs zwischen NSDeutschland und der Schweiz für den Untersuchungszeitraum 1933 bis 1945 ein Forschungsdesiderat.

\section{Theaterpolitik und Theaterpraxis, 1933-1945}

Zur Theaterpolitik und Theaterpraxis im Nationalsozialismus sind bereits einige historische Überblickswerke erschienen. Dazu zählen jene von Günther Rühle ${ }^{73}$ über die NS-Theatergeschichte und das Exil oder die umfangreiche Darstellung zur Spielplanstruktur und NS-Dramatik im „Dritten Reich“, welche von Henning Rischbieter herausgegeben wurde. ${ }^{74}$ Zur NS-Theaterpolitik erschienen darüber hinaus zahlreiche Einzelstudien, wie Bärbel Schraders Darstellung der „Reichskulturkammer“ oder Steffan Hüppings Monografie über den Reichsdramaturgen Rainer Schlösser (1899-1945). ${ }^{75}$ Björn Weigel befasste sich mit dem sukzessiven Berufsverbot jüdischer Theaterunternehmer in Berlin und Stefan Dussel mit der „gleichgeschalteten“ Besucherorganisation unter Mitwirkung der „N.S.-Kulturgemeinde“ ${ }^{76}$ Zur Berliner Theaterstadt im Nationalsozialismus forschte Elisabeth Schulz Hostetter und berücksichtige dabei sowohl den Verwaltungsapparat der NSDAP als auch ausgewählte Theateraufführungen sowie kritische Stimmen aus der Zeit. ${ }^{77}$ Zudem erschienen Monografien über Berühmtheiten wie den Intendanten und Schauspieler Gustav Gründgens (1899-1963) ${ }^{78}$, über Heinz Hilpert $(1890-1967)^{79}$, Regisseur und Intendant des „Deutschen Theaters“ (1934-1938) in Berlin sowie Direktor des Wiener Theaters in der Josefstadt (1938-1945), oder über den NS-Dramatiker Hanns Johst $(1890-1978)^{80}$. Zahlreiche Forschungen

73 Vgl. Rühle 1974, Rühle 2007.

74 Vgl. Eicher und Panse 2000. Gesamtdarstellungen zur NS-Theaterpolitik vgl. u.a. Wardetzky 1983, Drewniak 1983a, Dussel 1988. Neuere Forschungen, vgl. Dalinger und Zangl 2018.

75 Vgl. Schrader 2008, Hüpping 2012.

76 Vgl. Weigel 2017, Dussel 2017.

77 Vgl. Schulz Hostetter 2004. Ferner zur NS-Theaterpolitik, 1933-1945, vgl. London 2000, Balfour 2001.

78 Zur Biografie, vgl. u.a. Blubacher 1999. Zum Verhältnis zwischen Gründgens, Hilpert und Göring vgl. In der Diktatur 1933-1945. In: Rühle 2007, 725-994.

79 Zur Person vgl. Blubacher 2005a. Vgl. Dillmann und Akademie 1990, Peter 2010.

$80 \mathrm{Zu}$ Person und Werk vgl. Düsterberg 2004. 
sind zu Orchestern, Opern, Operetten, Tanz, Kabarett, Film, Literatur ${ }^{81}$, Rundfunksendungen und Kunst im Nationalsozialismus entstanden. ${ }^{82}$

Bereits seit den 1970er Jahren befassen sich Untersuchungen wie jene Henning Eichenbergs, Rainer Stommers oder jüngst von Evelyn Annuß mit dem Eventcharakter der „Erlebnisgemeinschaft“ an NS-Massenspielen, wie zum Beispiel dem „NS-Thingspiel“, den „Arbeiterweihespielen“, den Aufführungen während der Olympiade 1936 in Berlin oder den Festspielen in der Schweiz. ${ }^{83}$ Annuß untersucht in ihrer Forschung die ästhetische Formgeschichte der populistischen Massenspiele im Nationalsozialismus und ihrem Fortleben bis in die Gegenwart. Im Rückgriff auf die Affect Studies stellt sie einen affektpolitischen Wandel von der chorischen Theaterform (mit Liturgie und Auftritt eines Kollektivs) hin zum Event mit der Formspezifika eines medialen Spektakelcharakters fest. Im Unterschied zur vorliegenden Untersuchung berücksichtigt Annuß die Ansätze der Gefühlsgeschichte nicht. Stattdessen bezieht sie sich auf die empirisch geprägten Affect Theories. Annuß verwendet zeitgenössische Begriffe wie „PR-Strategie“ und spricht von Affekten und dem Affektiven in Anlehnung an den Philosophen Gilles Deleuze „als unpersönlich und dividuell begriffen, als energetische Dimension des Dazwischen mithin und nicht als, wenn auch sozial konstruiertes, individuierbares Gefühl“ ${ }^{84}$ Statt Gefühle als historisch auftretend, dynamisch wandelbar und erlernbar zu begreifen und daher als einen spezifisch differenzierbaren Untersuchungsgegenstand zu analysieren, geht Annuß generalisierend von einer „Mobilisierung der

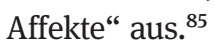

Die Schweizer Theatergeschichte, unter Berücksichtigung des Zeitraums von 1933 bis 1945, wurde im umfangsreichen Sammelband „Schweizertheater“ von den Herausgeber^innen Hans Amstutz, Ursula Käser-Leisibach und Martin Stern dargestellt. Darin führen die Autor`innen Forschungsdesiderate auf, von denen

81 Zur NS-Literaturpolitik und „deutschen Klassik“, vgl. u.a Denkler und Prümm 1976, Wulf 1983, Albert 1994a, Caemmerer 1996, Graeb-Könneker 2001, Barbian 2008, Ruppelt 2005, Sarkowicz und Mentzer 2011, Barbian 2013. S. Kap. 13.1.

82 Vgl. u. a. Bildende Kunst: Zuschlag 1995, Benz, Eckel und Nachama 2015; jüdisches Musik- und Theaterleben: Stompor 2001, Fritsch-Vivié 2013; Kabarett: Jelavich 1993; Film: O’Brien 2006; Musiktheater und Orchester (Oper, Operette, Sinfoniekonzerte): Fulfs 1995, Aster 2007, Schaller 2007, Nierenz 2010, Werr 2014, Dolaplis 2019; Rundfunk: Koch 2003, Sarkowicz 2004; Tanz: Manning 1993, Karina und Kant 1999, Karina und Kant 2003. Mehr Literaturhinweise zur Berliner Theaterstadt $1890-1945$ s. Kap. 9.

83 Vgl. Eichberg u. a. 1977, Stommer 1985, Engler und Kreis 1988. Neuere Studien zu Massenspiel und Festspiel vgl. Deutsches Schauspiel. Ein unpolitisches Theater? In: Reichel 2006, 435-447, Elfert 2009, Merkel 2014, Hoffmann-Allenspach 2018, Anne Keller 2018, Annuß 2019.

84 Vgl. Annuß 2019, 3, Fußnote 9.

85 Vgl. ebd., 3. 
viele bis heute noch nicht ausgeschöpft wurden. ${ }^{86}$ Fallstudien zum „Cabaret Cornichon“ oder zur „Freien Bühne“ sind vereinzelt erschienen ${ }^{87}$ oder sind, im Fall der Forschungsprojekte zur „Zürcher Theatergeschichte“ (Teil 1: „Opernhaus Zürich und das ,Dritte Reich““) ${ }^{88}$ sowie zum Regisseur und Mitbegründer der „Schweizer Gesellschaft für Innerschweizer Theaterkultur“ (gegr. 1927), Oskar Eberle (1902-1956), am Entstehen. ${ }^{89}$ Zum Schweizer Theaterexil, mit Fokus auf das Schauspielhaus Zürich ${ }^{90}$ und die restriktive Einwanderungspolitik während des Zweiten Weltkriegs, sind die transnational angelegten Untersuchungen von Ursula Amrein und Kristina Schulz zu nennen. ${ }^{91}$ Zukünftig wären weitere transnationale Forschungsperspektiven wünschenswert, die neben Zürich gesamtschweizerisch auch weitere deutschsprachige Theaterstädte wie Basel ${ }^{92}$, Bern $^{93}$, Luzern $^{94}$ oder St. Gallen und sprachübergreifend die Romandie (insbesondere Genf und Lausanne), das Tessin sowie die Nachbarländer Deutschland, Österreich, Frankreich und Italien miteinschließen würden.

\section{Forschungsdesiderat: Theaterwissenschaft und Arbeitspsychologie, 1933-1945}

Die transnationale Wissenszirkulation zwischen Theaterwissenschaft, Theaterpolitik, Theaterpraxis und Arbeitspsychologie stellt ein Forschungsdesiderat dar, dem sich die vorliegende Untersuchung am Beispiel der Theaterwissenschaftler Heinz Kindermann und Oskar Eberle sowie des Arbeitspsychologen und Theaterpraktikers Hendrik de Man annimmt (s. Kap. 15 und 16). Die transnational

86 Vgl. Künftige Aufgaben, 569-575. In: Stern 2000. Zur Schweizer Theatergeschichte vgl. Kotte, Gojan und Aguet 2005.

87 Vgl. Kotte 2002, Keller 2011.

88 Zum Forschungsprojekt (Leitung: Dr. Christian Mächler): https://www.theaterwissenschaft. unibe.ch/forschung/projekte/laufende_projekte/zuercher_theatergeschichte/index_ger. html\#e830185, 09.01.2020.

89 Zum Forschungsprojekt (Leitung: PD Dr. Heidy Greco-Kaufmann): https://www.theaterwissen schaft.unibe.ch/forschung/projekte/laufende_projekte/oskar_eberle_1902_1956/index_ger.html, 09.01.2020.

90 Zur Bedeutung des Schauspielhauses Zürich als Exiltheaterstadt vgl. Riess 1963, Bachmann und Schneider 1987, Amrein 1995, Lendenmann 1995, Kröger und Exinger 1998, Reichenbach und Lenherr 2006, Starz 2008, Hasler 2015.

91 Vgl. Amrein 2004, Amrein 2007, Amrein 2013; vgl. Schulz 2012; vgl. u. a. auch Bruns 2007.

92 Vgl. Blubacher 1995.

93 Vgl. Greco-Kaufmann 2017.

94 Vgl. Wüest 1990. 
vernetzte deutschsprachige NS-Theaterwissenschaft, NS-Dramatik ${ }^{95}$ sowie die politisch vielseitige Schweizer Dramenproduktion ${ }^{96}$ zur Zeit der „geistigen Landesverteidigung“ werden erst in den letzten Jahren von der deutschen und österreichischen Theaterwissenschaft berücksichtigt. ${ }^{97}$

Vereinzelt sind Fallstudien zu Theaterwissenschaftler`innen und Germanist`innen erschienen, welche an Lehrstühlen der Universitäten Berlin, Bonn, Frankfurt, Kiel, Köln, Jena, Leipzig ${ }^{98}$, München und Wien während der Weimarer Republik (Max Herrmann in Berlin u.a.) ${ }^{99}$ und noch während des Nationalsozialismus tätig waren, wie Julius Petersen (1878-1941) in Berlin, Carl Niessen $(1890-1969)^{100}$ in Köln, Hans Knudsen $(1886-1971)^{101}$ in Berlin, Hugo Dinger $(1865-1941)^{102}$ in Jena und Artur Kutscher $(1878-1960)^{103}$ in München, oder finden in der Literatur zumindest Erwähnung. ${ }^{104}$ Hervorzuheben ist die Forschung von Hans-Harald Müller und Mirko Nottscheid zur „Berliner Gesellschaft für Deutsche Literatur“ (1888-1938) und deren untersuchten Verbindung zur „Gesellschaft für Theatergeschichte“ (gegr. 1902) unter der Ära Max Herrmanns (1916-1938). ${ }^{105}$ Die Theaterwissenschaftlerin Birgit Peter hat 2008 damit begonnen, das transnationale Netzwerk der NS-Theaterwissenschaft nach dem „Anschluss“ Österreichs 1938 und der damit einhergehenden Gründung des „Zentralinstituts für Theaterwissenschaft“ 1943 in Wien systematisch aufzuarbeiten. ${ }^{106}$

95 Vgl. Ketelsen 1968, Düsterberg 2009.

96 Zum „völkischen“ Theater in der Schweiz vgl. Hodler 2016. Zur Transnationalität Schweizer Dramen im Nationalsozialismus vgl. ebd., Caluori 1996. Verzeichnis Schweizer Bühnenwerke 1900 - 1952, vgl. Kotte, Gojan und Aguet 2005.

97 Frühe Problematisierungen der Wissenschaftsgeschichte der NS-Theaterwissenschaft, vgl. u. a. Wulf 1964, Münz 1974, Wicclair 1989, Girshausen 1990, Fischer-Lichte 1994. Zur Geschichte der Theaterwissenschaft vgl. Klier 1981, Balme 2010.

98 Vgl. Kirschstein 2009.

99 Berthold Litzmann (1857-1926) in Bonn, Albert Köster (1862-1924) in Leipzig, Eugen Wolff (1863-1929) in Kiel. Vgl. Fischer-Lichte 1994, 13. Zu Herrmann, vgl. Corssen 1998, Hollender 2013, Dörschel und Warstat 2018.

100 Vgl. Elvert 2008, Ellrich 2009. Vgl. Dissertationsprojekt (2012-2019) von Nora Probst, Universität Köln: „Objekte, die die Welt bedeuten. Carl Niessen und die Geschichte der Kölner Theaterwissenschaft.“ (erscheint voraussichtlich 2021 im Metzler Verlag).

101 Vgl. Lazardzig 2018.

102 Vgl. Schmitt 2002.

103 Vgl. Buglioni 2016.

104 Vgl. Hausmann 2011. Vgl. Annuß 2019, 59, Fußnote 149. Vgl. Primavesi 2020.

105 Vgl. Müller und Nottscheid 2011.

106 Vgl. Peter und Payr 2008. Zur Fachgeschichte der Theaterwissenschaft vgl. u. a. Wulf 1964, Corssen 1998, Herrmann 2005; vgl. Hulfeld 2007, Engelhart 2008, Hulfeld und Peter 2009, Peter 
Zur Literaturwissenschaft im Nationalsozialismus und während der Zeit der „geistigen Landesverteidigung“ erschienen hingegen bereits Überblicks- und Einzelstudien, wie die Untersuchungen der Germanistinnen Gerhard Kaiser (1927-2012), Beatrice Sandberg und Julian Schütt zeigen. ${ }^{107}$

Ein wissenschaftshistorisches Überblickswerk, welches das transnationale Netzwerk der deutschsprachigen Theaterwissenschaft im Rahmen ihrer akademischen Institutionalisierungsprozesse aufzeigen würde, fehlt bis heute. Transnational zu erforschen wären universitäre Organisationen (Institutsgründungen, Gesellschaften, Vereine, Interessenverbände), deren vernetzte Akteur^innen (Professor^innen, Lehrbeauftragte, Assistent*innen, Doktorierende, Studierende), deren Wissensproduktion (Forschung und Lehre), Sammlungstätigkeit (Theatersammlungen der Universitäten in Berlin, Wien ${ }^{108}$, Köln ${ }^{109}$ u.a.) und Vermittlungsarbeit (Ausstellungen, Zeitschriften, Vorträge, Kongresse). Eine solche Analyse müsste im wissenshistorischen Kontext der spezifischen nationalstaatlich propagierten Bildungs-, Hochschul- und Kulturpolitik sowie mit Blick auf das vielseitige Theaterwesen (Laien- und Berufstheater: Intendant*innen, Drama-

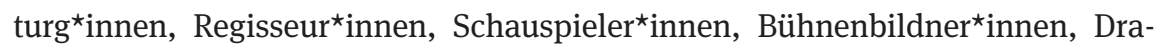
matiker^innen, Publikum) geschehen.

Die wissenschaftshistorische Fachgeschichte der Theaterwissenschaft in der Schweiz ist nach wie vor ein Forschungsdesiderat (darauf wird in Kap. 15.6 Bezug genommen). ${ }^{110}$ Die Schweizer Theatergeschichte wird von der Geschichtsforschung seit dem Abschlussbericht der „Unabhängigen Expertenkommission Schweiz - Zweiter Weltkrieg“ (UEK) (1996-2002) wieder marginalisiert. ${ }^{111}$ Das schweizweit einzige Institut für Theaterwissenschaft, das 1992

2009, Nieß 2010, Hochholdinger-Reiterer 2011, Peter 2013, Cuba 2017, Probst 2018, Dörschel und Warstat 2018, Lazardzig 2018, Probst 2020a, Probst 2020b, Probst (im Druck).

107 Vgl. Schütt 1996, Sandberg 2007, Kaiser 2008; vgl. u. a. auch Dainat und Danneberg 2003. $108 \mathrm{Zu}$ Wien vgl. Peter 2012, vgl. Cuba 2018.

109 Zu Köln vgl. Forschungsprojekt (2017-): „(Re-)Collecting Theatre History“ . BMBF-Verbundprojekt der Theaterwissenschaftlichen Sammlung unter der Leitung von Professor Dr. Peter W. Marx, Koordination Dr. Nora Probst zusammen mit den Theaterhistorischen Sammlungen des Instituts für Theaterwissenschaft, FU Berlin sowie dem Center for eHumanities der Universität zu Köln, https://phil-fak.uni-koeln.de/36551.html, 09.01.2020.

110 Vgl. Haffter 2021 (im Druck). Zur Institutsgründung vgl. Marinucci 2018.

111 Die UEK konzertierte sich vor allem auf politische, militärische und wirtschaftliche Aspekte der Schweizer Geschichte während des Zweiten Weltkriegs. Beeinflusst von der UEK entstanden aber in dieser Zeit einige aufschlussreiche theaterhistorische Untersuchungen, vgl. u.a. Engler und Kreis 1988, Marchal und Mattioli 1992, Kreis 1995, Clavien und Oltramare 1995, Mooser 1997, Amstutz 1997, Amstutz, Käser-Leisibach und Stern 2000. Zur UEK: https://www.uek.ch/de/, 14.12. 2020. 
an der Universität Bern gegründet wurde, wird sich unter der Leitung von Prof. Dr. Beate Hochholdinger-Reiterer in den nächsten Jahren diesem Forschungsdesiderat zuwenden. ${ }^{112}$

112 Zur Theaterwissenschaft in der Schweiz vgl. Engler 1990, Hochholdinger-Reiterer 2015, dies. 2016. 